\title{
Ciliary beat frequency in newborn infants
}

\author{
C O’Callaghan, K Smith, M Wilkinson, D Morgan, K Priftis
}

\begin{abstract}
The ciliary beat frequencies of $\mathbf{3 1}$ newborns and 14 adults were measured in vitro by a photodiode method at body temperature $\left(37^{\circ} \mathrm{C}\right)$. The mean (SD) neonatal ciliary beat frequency $(12.7(0.82) \mathrm{Hz})$ was faster than that in adults $(11.1(0.84) \mathbf{H z})(\mathbf{p}<0.01)$. Increased ciliary beat frequency may be advantageous during the neonatal period.
\end{abstract}

Ciliary dyskinesia should be considered in any neonatal patient or infant with unexplained respiratory distress. Buchdahl showed that when a child's respiratory problems are linked to ciliary dyskinesia, respiratory symptoms often start in the perinatal period. ${ }^{1}$ Ciliary problems can be diagnosed by structural or functional studies.

Ciliary ultrastructural examination may, however, be normal despite impaired function as defined by a reduction in ciliary beat frequency. The saccharin test of ciliary function requires cooperation and can not be carried out in young children, ${ }^{2}$ so in vitro measurement of ciliary beat frequency seems to be the most effective screening procedure for ciliary function in this age group. A reference range for ciliary beat frequency had not been defined for children.

The aims of our study were to determine a reference range of ciliary beat frequency in newborns, and to compare this with adult values.

\section{Patients and methods}

Thirty well newborn children less than 5 days of age (13 girls and 17 boys) were studied between September 1989 and February 1990. Fourteen adult volunteers mean (SD) age 21 years who had not had an upper respiratory tract infection for at least six weeks were also studied. All were non-smokers and none had a history of chest disease.

A sample of ciliated epithelium was obtained from the inferior turbinate of the nose with a cytology brush without anaesthesia. Cilia were suspended in $1 \mathrm{ml}$ of medium 199 cell culture fluid and a sample of this examined in a sealed microscope slide cover slip chamber. The preparation was placed on a heated microscope stage maintained at $37^{\circ} \mathrm{C}$ and allowed to equilibrate for 10 minutes. Specimens were examined with an inverted phase contrast microscope (Leitz-Vetzlor Orthoplan) at a magnification of 100 with oil immersion. A video camera (Hitachi QP-140E/K) projected the image on to a video display unit. The resulting magnification was $\times 2000$. A video enhancer circuit was added to the circuit to accentuate ciliary outlines.

Ciliary beat frequency was measured by holding a PIN photodiode probe over the moving ciliary edge. Fluctuations in intensity from the moving cilia were converted, amplified, and filtered to generate a voltage wave form that was displayed on the screen of a digital storage oscilloscope using a method described by Teichtal et al. ${ }^{3}$ The wave forms captured were plotted on to a chart recorder. The ciliary beat frequencies from at least 10 separate cilial edges were recorded from each patient.

To assess the variability with time, nine newborn infants had further biopsies 24 hours later and the ciliary beat frequency measured again. The observer was unaware of the source of the second sample.

\section{CALIBRATION OF THE PHOTODIODE}

To calibrate the PIN photodiode amplifier and recording system, a BBC microcomputer program was written to produce a flashing cursor on the video monitor. The program could produce a range of frequencies from 2.5 to $35 \mathrm{~Hz}$ by varying the time for which the flashing cursor was on or off in integer steps of $0 \cdot 1$ seconds. These frequencies were measured by the photodiode system and the results obtained compared with the frequency generated by the microcomputer.

\section{STATISTICAL ANALYSIS}

The unpaired Student's $t$ test was used to compared the results of adult and neonatal ciliary beat frequencies. An intraclass correlation coefficient was calculated to assess reproducibility. ${ }^{4}$

Ethical approval was obtained from the Nottingham Hospitals ethics committee.

\section{Results}

The mean (SD) ciliary beat frequency was $12 \cdot 7$ $(0.82) \mathrm{Hz}$ for the neonatal group and $11 \cdot 1(0.84)$ $\mathrm{Hz}$ for the adult group ( $<<0.01$ ) (figure). The intraclass correlation coefficient was 0.85 $(\mathrm{p}<0.002)$ between samples taken from the nine neonatal patients on two consecutive days. An intraclass correlation coefficient of 0.99 $(\mathrm{p}<0.0001)$ was obtained when the signal from the BBC computer was compared with the frequency detected by the photodiode system. 


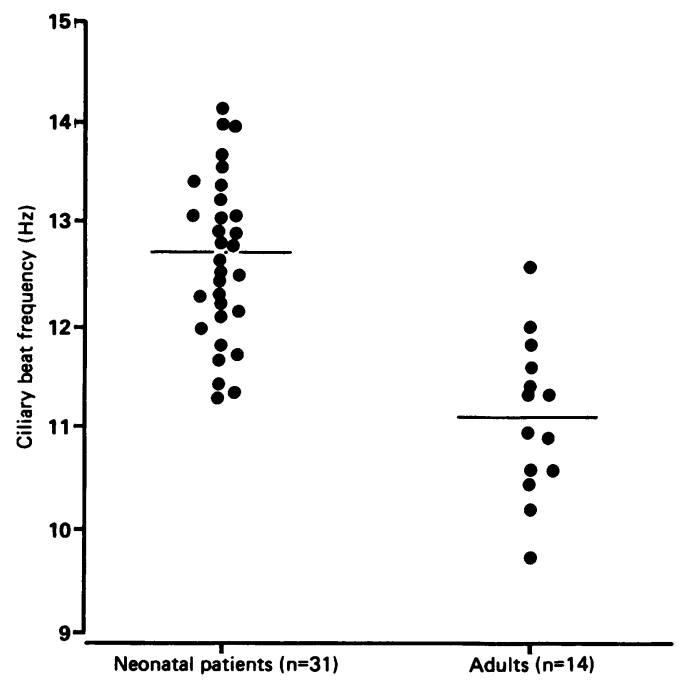

Ciliary beat frequency in 14 healthy adults and 31 newborm infants.

\section{Discussion}

When examined at body temperature the cilia of newborn infants had a significantly higher beat frequency than those of adult volunteers. This increased frequency may aid mucus clearance in newborn infants and therefore be advantageous.

The photodiode system accurately recorded frequencies from 2.5 to $35 \mathrm{~Hz}$. The high intraclass correlation coefficient suggests good reproducibility in measurements of ciliary beat frequency when cilia from the same infant were studied on consecutive days.
It is difficult to explain increased ciliary beat frequency by changes in ciliary ultrastructure, as a previous study showed no ultrastructural differences between neonatal and adult cilia on electron microscopy. ${ }^{5}$ This suggests that a difference in metabolism may account for the faster neonatal ciliary beat frequency.

Buchdahl et al presented strong evidence that children with unexplained chronic respiratory disease, in particular those with symptoms starting in the perinatal period, should be investigated for ciliary dyskinesia. ${ }^{1}$ In vitro testing of ciliary beat frequency seems to be the best screening test for ciliary abnormality in this age group. Our results show that it is not possible to extrapolate results of adult ciliary function studies to the neonatal period, however, which confirms the need for separate laboratories to establish their own reference ranges for ciliary beat frequencies at various ages.

We would like to thank Nottingham Hospital trustees for financial help.

1 Buchdahl RM, Reiser J, Ingram D, Rutman A, Cole PI, Warner JO. Ciliary abnormalities in respiratory disease. Warner J Jis Child 1988;63:238-43.

2 Greenstone M, Cole PJ. Ciliary function in health and disease. BrF Dis Chest 1985;79:9-26.

3 Teichtal H, Wright PL, Kirsner RCG. Measurement of invitro cilial beat frequency: a television video modification of the transmitted light technique. Med Biol Eng Comput 1986;24:193-6.

4 Lee J, Koh D, Ong CN. Statistical evaluation of agreement between two methods for measuring a quantitative variable. Comput Biol Med 1989;19:61-70.

5 Barlow J, Wilkinson M, O'Callaghan C. Neonatal cilia: ultrastructure. Arch Dis Child 1990;65:708-10. 